\title{
EDUCACIÓN PARA LA PREVENCIÓN DE ENFERMEDAD CARDIOVASCULAR EN TRABAJADORES DE UNA INSTITUCIÓN DE EDUCACIÓN SUPERIOR ¡Evitando la ausencia laboral!
}

\begin{abstract}
Education for Prevention of Cardiovascular Diseases in a Higher Education Institution Workers: ¡Avoiding absence from work!
\end{abstract}

\author{
ISAbel Cristina Rojas Padilla, Yury Vergara López \\ Escuela Nacional del Deporte, Colombia
}

\section{KEY WORDS}

Risk Factors

Cardiovascular Disease

Promotion

Prevention

PALABRAS CLAVE

Factores de riesgo

Enfermedad cardiovascular

Promoción

Prevención

\section{ABSTRACT}

Cardiovascular diseases (CVD) are the cause of many deaths worldwide and the most paradoxical issue is that most of them can be prevented by acting on behavioural risk factors such as sedentary lifestyle, excess weight, inadequate diets, cigarette consumption and alcoholic beverages. Cardiovascular risk must be detected early to intervene it first and prevent it becomes a diagnosed disease. Promotion and prevention of healthy lifestyle habits prevent suffering and improve the quality of people's life, even more in active working individuals who must interact in a social environment daily.

\section{RESUMEN}

Las enfermedades cardiovasculares (ECV) son las causantes de muchas muertes a nivel mundial y lo paradójico es que la mayoría de ellas pueden prevenirse actuando sobre factores de riesgo comportamentales como sedentarismo, exceso de peso, dietas inadecuadas, consumo de cigarrillo y bebidas alcohólicas. El riesgo cardiovascular se debe detectar tempranamente para intervenirlo de manera precoz y evitar que se convierta en enfermedad diagnosticada. La promoción y prevención de hábitos de vida saludable disminuye padecimientos y mejora la calidad de vida de las personas, más aún en aquellos individuos laboralmente activos que deben interactuar en un ambiente social a diario.

Recibido: 24/11/2018

Aceptado: 01/11/2019

\section{GLOBAL KNOWLEDGE}




\section{Introducción}

L as enfermedades crónicas no transmisibles se han convertido en el día a día de la población mundial, inundando diagnósticos de personas de diferentes áreas, etnias, géneros, estratos sociales, labores, nivel educativo e inclusive edades, referente a los cuales, en el pasado se sobrecargaba en los adultos mayores. La Organización Mundial de la Salud (OMS) y la Organización Panamericana de la Salud (OPS) vienen realizando estudios enfáticos a nivel epidemiológico sobre patologías de alta incidencia, que prevalecen y crecen dentro de la población mundial y que no solo se relacionan con factores genéticos y microbianos, sino que están enmarcados en el nuevo estilo de vida de las personas, el cual se ve influenciado por la modernidad, la globalización y todos aquellos cambios que traen consigo estas fenomenologías, entre ellos el estrés, el tiempo, la alimentación, las ocupaciones, la participación social y las emociones negativas (Albalo J., 2016).

En cuanto a datos y cifras publicadas por la OMS en el 2015, las enfermedades no transmisibles (ENT) matan a 38 millones de personas cada año. 16 millones de las muertes atribuidas a las ENT se producen en personas menores de 70 años de edad; el $82 \%$ de estas muertes «prematuras» ocurren en países de ingresos bajos y medianos. Además, las enfermedades cardiovasculares constituyen la mayoría de las defunciones por ENT, 17,5 millones cada año, seguidas del cáncer $(8,2$ millones), las enfermedades respiratorias (4 millones), y la diabetes (1,5 millones) (OMS, 2015). Por otra parte, el consumo de tabaco, la inactividad física, el uso nocivo del alcohol y las dietas malsanas aumentan el riesgo de morir a causa de una de las ENT y son responsables de alrededor del $82 \%$ de las muertes por ENT (OMS, 2015).

La promoción de la salud genera a nivel de desarrollo, indicadores claves para trabajar en entornos laborales saludables puesto que el lugar de trabajo es donde las personas pasan la mayoría de las horas de sus días de semana, aspecto que realza la importancia y necesidad de crear ambientes laborales saludables. Un lugar de trabajo agradable debe promover una buena salud y este es un aspecto primordial para el desarrollo tanto personal como social y económico.

\section{Justificación}

Las enfermedades crónicas no transmisibles son un problema de salud pública a nivel mundial, responsables de un alto índice de muerte y que están demandando la detección de factores de riesgo con el fin de trabajar en la promoción y prevención disminuyendo así la prevalencia de este tipo de patologías. La carga patológica que implica el riesgo y más aun la ECV genera no solo un deterioro en la salud de la persona afectada sino un desbalance a nivel de todas aquellas actividades que esta persona realiza, considerando entre estas su contexto social, familiar y laboral. Una persona enferma no rinde en su trabajo de la misma manera que una quien goce de buena salud, a lo que se puede sumar la ausencia laboral por incapacidades, factor que incrementa gastos para el empleador por contratar reemplazos y pagar incapacidades y demás costos que puedan resultar.

Con la estrategia se busca aportar un nuevo enfoque de trabajo para toda la población, tanto quien padece riesgo cardiovascular como quienes están sanos o diagnosticados. Se busca una trascendencia en el tiempo teniendo en cuenta que el enfoque se direcciona hacia la prevención del riesgo y contribuir de esta manera a solucionar un problema que afecta el $50 \%$ de la población, cifra obtenida en un resultado preliminar de una investigación realizada por las mismas autoras de este escrito. Este es el motivo más relevante para iniciar el programa "Vida saludable y físicamente activa en el trabajo" la cual permitirá a la comunidad educativa comprender la importancia de reducir factores de riesgo cardiovascular y fortalecer todos aquellos que se consideren protectores y que beneficiarán no solo a cada individuo sino a sus familias y a la institución en la cual laboran, esto por medio de la educación basada en estilos de vida saludable.

\section{Antecedentes}

Bien es cierto que el riesgo cardiovascular es un problema alrededor de mundo, lo importante de este asunto es como las estancias involucradas están enfrentando este fenómeno. Ciertamente, la implementación de hábitos de vida saludable debería ser iniciativa personal, sin embargo, en 
muchas ocasiones es necesaria la intervención del entorno para lograr atraer el interés que esto merece. A nivel internacional, un estudio conducido en Brasil (Casas S.B. \& Klijn T.P., 2006) llamado "Promoción De La Salud Y Un Entorno Laboral Saludable" cuyo objetivo fue generar un entorno laboral saludable, promovió la participación de todos los actores para controlar, mejorar y mantener la salud y el bienestar de los trabajadores reorientando todos aquellos servicios de salud incluyendo promoción y sus aspectos relacionados teniendo en cuenta que sus trabajadores pudieran acceder a los servicios de salud primaria, preventiva y ocupacional y así cumplir su objetivo ya que una buena salud en el entorno laboral es primordial para el desarrollo y se encamina a buscar protección de la salud de trabajadores y grupos familiares. En Colombia, el proyecto "Educar Para La Salud En Educación Superior: Tendencias Y Retos" (Meda R., De Santos F., Palomera A. \& Del Toro R., 2012) se planteó el propósito de impulsar la promoción de la salud con el fin de promover y proteger la salud de su comunidad con diferentes programas como Universidad saludable, Universidad libre de humo de tabaco, Programa Eco-CUCs y el programa de Actividad física y salud. Los logros de esta estrategia fueron cambio de actitudes, conductas, patrones y hábitos nocivos para la salud además de la promoción de beneficios de una alimentación saludable y la concientización de la influencia de estos hábitos en el bienestar físico, mental y emocional en los miembros de la comunidad.

En línea con estas dos estrategias mencionadas anteriormente se plantea el programa "Vida saludable y físicamente activa en el trabajo" basada en hallazgos de una investigación con la misma población a intervenir constituida por adultos entre los $22 \mathrm{y}$ 64 años de edad laboralmente activa una la institución de educación superior Colombiana en la cual se encontró prevalencia de un $50 \%$ de presencia de riesgo cardiovascular determinada por la media (49.5 \pm 6 ), cifra muy alarmante y que debe demandar toda la atención y cuidado en pro de preservar la salud de los trabajadores, esto en miras a promover estilos de vida saludable y evitar la ausencia laboral por complicaciones de salud, especialmente cardiovasculares.

\section{Marco referencial}

El desarrollo de patrones de hábitos de vida saludables tiene que ver con la promoción de la salud, direccionado hacia un nivel de vida con excelentes condiciones de alimentación, de práctica de actividad física regular, oferta laboral, posibilidad de educación y estrategias para el descanso y la recreación. Todo esto influye positivamente en la salud evitando el desarrollo posterior de enfermedades crónicas, por lo cual es tan importante contextualizar hábitos y estilos de vida además de las ENT y en especial la ECV y su riesgo.

De esta manera, los hábitos conforman las costumbres, actitudes y comportamientos de las personas, las cuales conllevan a formar $y$ consolidar pautas, conductas y aprendizajes que se mantienen en el tiempo y repercuten (favorable o desfavorablemente) en el estado de salud, nutrición y bienestar (Diaz L. K., Casa D.s, \& Ortega F., 2013).

\section{Hábitos alimentarios}

Los hábitos implican cierto automatismo determinando en muchas ocasiones el modo de actuar de los individuos, al igual que sus preferencias y elecciones (Bourges H., 1988) En cuanto a las poblaciones, estos son la expresión de creencias y tradiciones y están ligados al medio geográfico y a la disponibilidad alimentaria. Por su parte, los hábitos alimentarios del mundo occidental se caracterizan, cuantitativamente, por un consumo excesivo de alimentos que superan las ingestas recomendadas en cuanto a energía y nutrientes y cualitativamente, por un tipo de dieta rica en proteínas y grasas de origen animal. Además de una alimentación correcta y equilibrada, la lucha contra el sedentarismo y el impulso de estilos de vida que incluyan una dedicación al ejercicio físico son la mejor manera de mantener niveles adecuados de salud en las distintas etapas de la vida (Ministerio del Interior, Educación cultura y sanidad, 2009).

\section{Estilo de vida saludable}

Son comportamientos o factores protectores de la calidad de vida, que al asumirlos responsablemente ayudan a prevenir desajustes 
biopsicosociales y mantener el bienestar para generar calidad de vida, satisfacción de necesidades y desarrollo humano (Diaz L. K., Casa D.s, \& Ortega F., 2013).

\section{Enfermedades crónicas no transmisibles}

Las enfermedades crónicas no transmisibles son enfermedades de curso prolongando que necesitan tratamientos continuos para su control. No se resuelven espontáneamente y rara vez se logra una cura completa, (Escobar M.C. et al, 2000) las definen y las enumeran como: enfermedades cardiovasculares, ataques al corazón y accidentes vasculares cerebrales, hipertensión arterial, artritis, cáncer y enfermedad pulmonar crónica; enfermedades neurológicas crónicas, diabetes, insuficiencia renal y la obesidad.

Por su parte, la obesidad se ha considerado enfermedad crónica, ya que es una afección de larga duración y que puede ser de progresión lenta, cuyo signo inicial es el exceso de peso que al no controlarse se convierte en tal patología crónica. Frente a esto, en Colombia la Ley 1355 de 2009 define la obesidad y las enfermedades crónicas no transmisibles asociadas a esta como una prioridad de salud pública y se adoptan medidas para su control, atención y prevención. El sobrepeso y la obesidad se definen como una acumulación anormal o excesiva de grasa que puede ser perjudicial para la salud.

Las ENT se deben en gran medida a cuatro factores de riesgo comportamentales que se han afianzado de forma generalizada como parte de la transición económica, los rápidos procesos de urbanización y los modos de vida del siglo XXI: el consumo de tabaco, las dietas malsanas, la inactividad física y el uso nocivo del alcohol. (OMS, P. 5, 2010)

Desde el área de nutrición y considerando uno de los cuatro factores de riesgo de las ECNT, la dieta, es posible considerarla como factor de riesgo, pero también como factor protector puesto que puede ser de gran ayuda en la prevención y tratamiento de cada una de las patologías. Diferentes investigaciones aseguran que una dieta que sea suficiente, accesible y variada reduce en gran medida el riesgo de padecer alguna de las ECNT (Fundación B, 2008). Por ejemplo, el cáncer se reduce con una dieta rica en macronutrientes (Fundación B, 2008), resaltando las propiedades antioxidantes y los beneficios en la respuesta inflamatoria o inmune en el crecimiento de células y reparación del ADN (Romieu I., 2011). En lo que respecta a la EPOC, su relación más marcada a lo largo de la historia ha sido el consumo de cigarrillo, sin embargo, investigaciones afirman que un tercio de las personas diagnosticadas con EPOC nunca han fumado, así que otros factores pueden intervenir en el desarrollo de esta enfermedad como por ejemplo "una dieta sana, baja en carne roja y rica en granos integrales podría reducir el riesgo de contraer la enfermedad; la hipótesis de los autores del estudio es que los antioxidantes en una dieta saludable serían los responsables de este efecto protector frente al EPOC"(Varraso R. et al, 2010).

Por otra parte, no solo la dieta influye como factor de riesgo, pues la actividad física reduce el riesgo a padecer muchas enfermedades como la obesidad, la hipertensión arterial, diabetes, entre otras (Coitinho D., Monteiro C., \& Popkin B., 2002).

La práctica de actividad física de forma regular debería ser una constante a lo largo de la vida de todos los individuos, ya que se han comprobado desde diferentes puntos de vista los beneficios de ésta sobre la salud, reducir riesgos de sufrir enfermedades como: diabetes tipo 2, hipertensión arterial, colesterol, artritis, problemas cardiovasculares, etc. Sin embargo, los estudios sobre hábitos muestran una tendencia de la población hacia la adquisición de estilos de vida sedentarios y poco saludables, entendiendo como sedentarismo la falta de actividad física regular, definida como: "menos de 30 minutos diarios de ejercicio regular y menos de 3 días a la semana" (OMS, 2015).

\section{Enfermedades cardiovasculares}

Las enfermedades cardiovasculares son trastornos del corazón y los vasos sanguíneos. Las principales causas de enfermedad vascular son el consumo de tabaco, la falta de actividad física y una alimentación poco saludable (OMS, 2008) que constituyen una de las causas más importantes de discapacidad y muerte prematura en todo el mundo. Los problemas subyacentes son aterosclerosis, episodios coronarios (infarto de miocardio) $y$ 
cerebrovasculares (ataque apopléjico). La modificación de los factores de riesgo tanto alimentarios como de actividad física, puede reducir los episodios cardiovasculares y la muerte prematura tanto en las personas con enfermedad cardiovascular establecida como en aquellas con alto riesgo cardiovascular debido a uno o más factores de riesgo (OMS, 2015).

\section{Programa}

El programa "Vida saludable y físicamente activa en el trabajo" tiene como objetivo el potencializar el ejercicio físico, la sana alimentación y la disminución de hábitos nocivos para la salud como factores protectores para enfermedad y/o riesgo cardiovascular en la población trabajadora de la institución. El alcance de esta estrategia es únicamente desde el factor educativo, es decir no se realizarán programas de diagnóstico ni intervención.

\section{Metodología}

\section{Población}

La población objeto de la estrategia educativa está constituida por adultos entre los 22 y 64 años, colombianos, laboralmente activos y vinculados por contrato con la institución. Están divididos en tres grupos: el primero compuesto por personal administrativo, quienes trabajan en horarios de $8 \mathrm{am}$ a $12 \mathrm{~m}$ y de $2 \mathrm{pm}$ a $6 \mathrm{pm}$ en oficina. El segundo lo compone personal de servicios generales con horarios diversos generalmente entre las $6 \mathrm{am}$ y las $3 \mathrm{pm}$ con una hora de almuerzo y quienes tienen labores que implican movimiento constante. Por otro lado, está el grupo de docentes.

\section{Estrategias}

Las estrategias edu-comunicativas y de impacto visual simple planeadas para tal fin son:

1. "Encuentros educativos": esta estrategia reúne foros y conversatorios, liderados por una docente de nutrición encargada del tema alimenticio y por una licenciada en educación física encargada del tema de actividad física, cuyo objetivo se centra en encontrar de maneja conjunta y por juego de roles, la importancia de la práctica de ejercicio para la salud física y mental.

2. "Debates alimenticios" acerca de cómo debe ser la alimentación, tocando los términos de: adecuada, suficiente, completa, variada y equilibrada. Este debate es liderado por una docente de nutrición y su objetivo es hallar características propias de los diferentes momentos de comidas durante un día. Ejercicio realizado con ayuda de estudiantes del pregrado de nutrición de la institución y con el servicio de alimentación de la institución como escenario propicio para la actividad.

3. Jornada única de "actívate saludablemente", liderado por la docente de educación física y con el apoyo de estudiantes del pregrado de Profesional en Deporte, con el objetivo de indicar diversas formas de realizar actividad física dentro de la institución y en el tiempo libre $\mathrm{y}$ teniendo como escenarios los diferentes campos de la institución como el coliseo, las oficinas, los pasillos entre bloques y salones y las plazoletas.

4. "Pausas activas CV" con temas y ejercicios enfocados a la disminución del RCV con la intervención de estudiantes de la práctica del pregrado de fisioterapia de la institución y en los momentos destinados a pausas activas que se deben realizar en cada una de las dependencias de la Institución. Estas supervisadas por las docentes encargadas del programa.

5. Programa "Ayudo al planeta y subo escaleras" promoviendo el uso adecuado de ascensores, para personas con discapacidad de movilidad, adultos mayores, mujeres embarazadas, o personas con niños pequeños en brazos, con opción de acceso por rampas y/o escaleras a pisos superiores.

\section{Beneficios del programa}

Con este programa se pretende crear conciencia de la importancia del auto cuidado para la prevención del RCV, morbilidades y de muerte prematura disminuyendo entre otras cosas el ausentismo laboral debido a esta patología. Así mismo se pretende disminuir el diagnóstico encontrado del $50 \%$ de la población con presencia de riesgo cardiovascular. 
El tercer beneficio que planteamos es incentivar el trabajo interdisciplinar dentro de la institución con la integración en el programa de tres de los pregrados: Nutrición, Fisioterapia y Profesional en Deporte.
Por último contribuir a la promoción y prevención de la salud a nivel individual y colectivo al mismo tiempo que se genere un ambiente agradable en el trabajo. 


\section{Referencias}

Abalo, J. (2016). Enfermedades crónicas no transmisibles: Un abordaje desde los factores psicosociales. Salud \& Sociedad, 7(2).

Bourges H., (1988). Costumbres, prácticas y hábitos alimentarios: deseables e indeseables. Arch latinoam nutric, 38(3), pp. 766-779.

Casas S.B., Klijn T.P. (2006). Promoción de la salud y su entorno laboral saludable. Rev. Latino-am Enfermagem, 14(1), pp. 136-41.

Coitinho D., Monteiro C., Popkin B. (2002). What Brazil is doing to promote healthy diets and active lifestyles. Public health nutrition, 5(1), pp. 263-267.

Diaz, L. K., Casas, D., Ortega, F. (2013). Hábitos alimentarios y nivel de actividad física de los estudiantes de tercero a séptimo grado de dos colegios de Santiago de Cali: Colegio Luis Fernando Caicedo y Colegio Colombo Británico. Universidad del Valle.

Escobar, M. C. et al. (2000). Mitos sobre la prevención y el control de las enfermedades no transmisibles en América Latina. Salud Pública de México, 42(1), pp. 56-64.

Fundación Bengoa. (2008). Guías de alimentación para Venezuela. Disponible en: https://www.fundacionbengoa.org/publicaciones/guias_alimentacion_venezuela.asp

Ley 1355 de 2009

Meda R, De Santos F, Palomera A, Del Toro R. (2012). Educar para la salud en Educación Superior: Tendencias y retos. RevEducCienc Salud, 9(2). pp. 86-94.

Ministerios del Interior, de Educación y Cultura y de Sanidad y Consumo. España. (2009). Nutrición Saludable y Prevención de los Trastornos Alimentarios.

OMS. (2008). Prevención de las enfermedades no transmisibles en el lugar de trabajo a través del régimen alimentario y la actividad física Foro Económico Mundial.

- (2010). Informe sobre la situación mundial de las enfermedades.

- (2015). Enfermedades no transmisibles.

- (2015). Enfermedades no transmisibles [cited 2016 marzo 04 de 2016]. Available from: http://www.who.int/mediacentre/factsheets/fs355/es/.

- (2015). Obesidad y Sobrepeso [cited 2016 marzo 03 de 2016]. Nota descriptiva No. 311]. Available from: http://www.who.int/mediacentre/factsheets/fs311/es/

Romieu, I. (2011). Diet and breast cancer. Salud pública de México, 53(5), pp. 430-439.

Varraso R. et al. (2015). Alternate Healthy Eating Index 2010 and risk of chronic obstructive pulmonary disease among US women and men: prospective study, vol. 350.

Zhang Ya-Ping et al. (2014). CFTR as a Novel Target and Mechanism for High-Fructose Diet and Salt-Induced Hypertension. Arteriosclerosis, Thrombosis, and Vascular Biology, vol 34. 